\title{
A reference handbook for daily use in equine practice
}

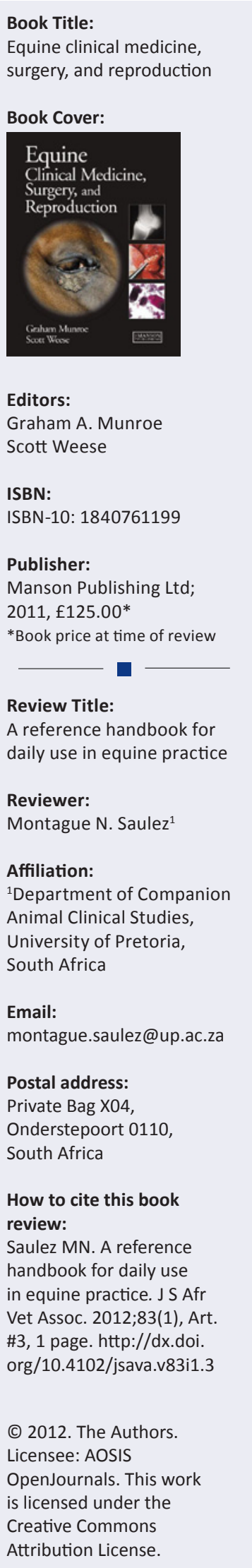

Numerous contributors have combined their expertise to produce a textbook encompassing a comprehensive range of topics in equine medicine, surgery and reproduction. As the editors have so aptly stated in their preface, it is virtually impossible to include all aspects of equine practice into a single volume. However, they should be commended on their efforts - this is a textbook that can be used daily in general equine practice, whether hospital-based or ambulatory.

This textbook comprises fifteen chapters, covering the musculoskeletal system, reproductive system, respiratory system, gastrointestinal system, liver, endocrine, urinary system, cardiovascular system, haemolymphatic system, nervous system, eyes, skin, wound management and infections of synovial structures, the foal, and behavioural problems. Within each chapter, diseases are classified together and presented in a repeatable and concise format, making it a pleasure to read and allowing quick access to information through a systems-based approach. In addition, there is an assortment of high-quality, full-colour images of clinical cases, including pertinent radiographic and ultrasound images. Indeed, these detailed images are what sets this book apart from the rest and will be of great benefit to the reader, assisting them in understanding the subject matter in a more thorough manner.

Having used this book for over a month now, myself, I feel it should be of great value to undergraduate veterinary students, interns and residents, as well as non-specialist equine practitioners. In fact, I highly doubt that this book will be left to sit around gathering dust for very long, for its versatility makes it an ideal reference guide for anyone involved in equine veterinary practice. 